\title{
Simulating Drug-Eluting Stents Progress Made and the Way Forward
}

\author{
Sean McGinty ${ }^{1}$, Christopher McCormick ${ }^{2}$, Sean McKee ${ }^{1}$, Marcus Wheel ${ }^{3}$, Simon Kennedy ${ }^{4}$ and \\ Keith Oldroyd ${ }^{5}$ \\ ${ }^{1}$ Department of Mathematics and Statistics, University of Strathclyde, Glasgow, UK. \\ ${ }^{2}$ Biomedical Engineering and Strathclyde Institute of Pharmacy and Biomedical Sciences, University of Strathclyde, \\ Glasgow, UK. \\ ${ }^{3}$ Department of Mechanical and Aerospace Engineering, University of Strathclyde, Glasgow, UK. \\ ${ }^{4}$ Institute of Cardiovascular and Medical Sciences, University of Glasgow, Glasgow, UK. \\ ${ }^{5}$ West of Scotland Regional Heart and Lung Centre, Golden Jubilee National Hospital, Glasgow, UK. \\ \{s.mcginty, christopher.mccormick, s.mckee,marcus.wheel\}@strath.ac.uk, Simon.Kennedy@glasgow.ac.uk, \\ keith.oldroyd@nhs.net
}

Keywords: Drug-Eluting Stents, Mathematical Modelling, Drug Release, Binding.

\begin{abstract}
Drug-eluting stents have significantly improved the treatment of coronary artery disease. Compared with their bare metal predecessors, they offer reduced rates of restenosis and thus represent the current gold standard in percutaneous coronary interventions. Drug-eluting stents have been around for over a decade, and while progress is continually being made, they are not suitable in all patients and lesion types. Furthermore there are still real concerns over incomplete healing and late stent thrombosis. In this paper, some modelling approaches are reviewed and the future of modelling and simulation in this field is discussed.
\end{abstract}

\section{INTRODUCTION}

Coronary heart disease (CHD) is the main cause of death in developed countries (Murray and Lopez, 1997). Such is the extent of the condition, CHD is responsible for $18 \%$ of all deaths in the United States annually (Lloyde-Jones, 2010). In simple terms, CHD is caused by a blockage or interruption to blood flow and often results in heart attack. It is generally considered that this problem is the result of fatty deposits accumulating and lining the arterial walls over a period of many years (Beers, 2004). The fatty deposits are called atheroma and the process during which the atheroma accumulates is termed atherosclerosis $(\mathrm{Lu}-$ sis, 2000). If left untreated, this leads to episodes of angina. As well as restricting blood flow, the atherosclerotic plaque is also vulnerable to rupture, ultimately resulting in a heart attack.

Traditionally, by-pass surgery was the only available treatment option. However, in the majority of cases this has now been replaced by minimally invasive procedures such as the insertion of a small metallic cage called a stent into the occluded artery. When a stent is implanted into an artery, the endothelium is severely damaged. This provokes an inflam- matory response which leads to excessive proliferation and migration of smooth muscle cells (SMCs) towards the lumen. The result is restenosis - the renarrowing of the arterial wall. The introduction of drug-eluting stents (DESs) has significantly reduced the occurrence of in-stent restenosis (ISR), by releasing a drug to inhibit SMC proliferation.

Drug-eluting stents have undoubtedly significantly improved the treatment of coronary artery disease. Compared with their bare metal predecessors, they offer reduced rates of restenosis and thus represent the current gold standard in percutaneous coronary interventions. But despite being around for over a decade, and while progress is continually being made, they are still not suitable in all patients or lesion types. Furthermore, there are still concerns over incomplete healing of the artery and late stent thrombosis (LST), resulting in anti-platelet therapy being recommended for at least 12 months after implantation (Stefanini and Holmes, 2013). Substantial efforts are now dedicated towards the development of enhanced DESs and in this paper we summarise the modelling approaches currently adopted and suggest important considerations for future modelling and simulation in this field. 


\section{THE CHICKEN OR THE EGG DILEMMA}

What is the logical sequence of events? Stent design and then modelling of drug release and uptake into the arterial wall? Or is it the other way round? Presently, the stent manufacturers are predominantly concerned with mechanical integrity of the device and as such the stent design is usually the first consideration. The stent must be flexible and expandable and stay in situ after deployment. During the expansion process the stent should undergo minimum shortening and after implantation should conform to the natural geometry of the vessel without any unnatural straightening (Khan et al., 2012). Radial strength is another key component; without this the stent will collapse under the strain of the artery. Furthermore, the materials used must be biocompatible and must not fracture.

But it is no good having a stent which is mechanically sound but does not elute drug in a favourable fashion. The release of the drug must be controlled so that it elutes over a defined period of time and, furthermore, the drug concentration in the arterial wall should ideally be maintained between therapeutic and toxic levels over and beyond the period of release. Taking this into account, it would seem that the drug release and uptake is intrinsically linked to the stent design and so a fair argument could be made either way.

Ideally the stent should be optimised, both in terms of the mechanical design (material used, number and pattern of struts) as well as drug loading (type and mass of drug, coating technique) so that the required clinical outcomes are realised. This optimisation is further complicated by the fact that every patient is different; the lesions vary in size and composition as well as location in the arterial tree. Furthermore, some patients have other complications such as diabetes or hypertension. Thus a single optimised stent design is simply unrealistic, but it may well be possible to develop an optimised stent for a set of different situations.

Realising the importance of stent design, a number of authors have investigated various aspects. The influence of stent geometry on restenosis was investigated (Garasic et al., 2000) while the distribution of the stent struts has been experimentally studied (Hwang et al., 2001), with the authors concluding that the mere proximity of delivery device to tissue does not ensure adequate drug targeting. The effect of the number of struts and the ratio between the coated area was researched by Delfour et al., and they attempted to optimize the effect of the dose (Delfour et al., 2005). A mathematical model for the study of the me- chanical properties of endovascular stents in their expanded state has also been proposed (Tambaca et al., 2010). Three-dimensional models of stent expansion have been presented by, among others, (Zunino et al., 2009) and (Horner et al., 2010).

\section{THE EVOLUTION OF THE DRUG-ELUTING STENT}

Over the past decade DESs have evolved, and already third-generation DESs have been developed. Despite their differences and improvements, these devices typically all have three main components; the stent platform, the coating and the drug.

\subsection{First generation DESs}

The first-generation DESs Cypher (sirolimus-eluting stent; Cordis Corporation) and Taxus (paclitaxeleluting stent; Boston Scientific Corporation) comprised a stainless steel platform with a drug containing polymer coating attached to the stent struts ((Stefanini and Holmes, 2013), (Tzafriri et al., 2012)). The philosophy behind this design was to allow the drug to be released gradually so as to avoid toxic levels of drug initially, but also to permit sustained delivery over many weeks. The Cypher stent actually consists of three distinct layers; a base coat, a drug-filled middle layer and a drug-free topcoat. This design enhances the controlled nature of the release. While the polymers used in these first generation DESs are different, they are both non-erodible. The drugs used (sirolimus and paclitaxel) are both lipophilic and are able to inhibit SMC proliferation and migration.

\subsection{Second generation DESs}

The second-generation DESs Endeavor (zotarolimuseluting; Medtronic), ZoMaxx (zotarolimus-eluting; Abbott Laboratories), Promus (everolimus-eluting; Boston Scientific Corporation) and Xience V (everolimus eluting; Abbott Laboratories) attempted to improve the biocompatibility and reduce the incidence of thrombosis rate which was associated with first-generation DES. These stents were generally designed with thinner struts and utilised cobalt-chromium and platinum chromium platforms. A variety of multi-layer polymer combinations were used on these stents to attempt to control the release. Generally these stents have been shown to exhibit lower thrombosis rates compared with first generation DES (Khan et al., 2012). 


\subsection{Third generation DESs}

Since the polymer coating in the earlier DES has been associated with local vascular inflammatory reaction and potentially inducing late stent thrombosis, newer generation stents have focussed on biodegradable polymers (BioMatrix, Biosensors Inc and NEVO, Cordis Corporation, Johnson \& Johnson), where the polymer carries and controls the drug release and then erodes or vanishes, and also coatings which do not contain any polymer at all (Yukon, Translumina and BioFreedom, Biosensors Inc), with the drug being contained on a modified surface of the stent.

\section{MODELLING THE RELEASE OF DRUG FROM STENTS}

Most of the modelling of drug release from stents in the literature has thus far been concerned with first generation DESs. Drug release from these stents has been modelled as a diffusion dominated process (see for example (McGinty et al., 2011) and (Pontrelli and de Monte, 2010)), with the drug concentration in the polymer $C_{p}$ satisfying a diffusion equation with drug diffusion coefficient $D_{p}$. In one dimension this is simply

$$
\frac{\partial C_{p}}{\partial t}=D_{p} \frac{\partial^{2} C_{p}}{\partial x^{2}}
$$

For the case of in-vitro drug release a zero flux boundary condition is normally assumed at the impermeable stent and either an infinite sink or Robin type boundary condition at the interface with the release medium. Models such as these can admit analytical solutions and have shown favourable results when compared with in-vitro experimental data. Figure 1 displays a comparison between the model predicted cumulative percentage of drug released and the average of four experimental in-vitro release profiles from the Cypher stent (for more details see Section 6). These analytical solutions also allow the drug diffusion coefficient to be estimated via a best fitting process.

Several simplifying assumptions are usually made. Firstly, it is assumed that the device geometry is that of a thin film with no edge effects so that the modelling may be restricted to one dimension. The diffusion of the drug in the polymer is thus considered to be isotropic and it is usually assumed that the diffusion coefficient is independent of time, space and concentration. Furthermore, the initial drug concentration is usually taken to be uniform. However, in reality this is not always the case. For example, the polymer coating on the Cypher stent consists of three distinct layers; a base coat, a drug-filled middle layer

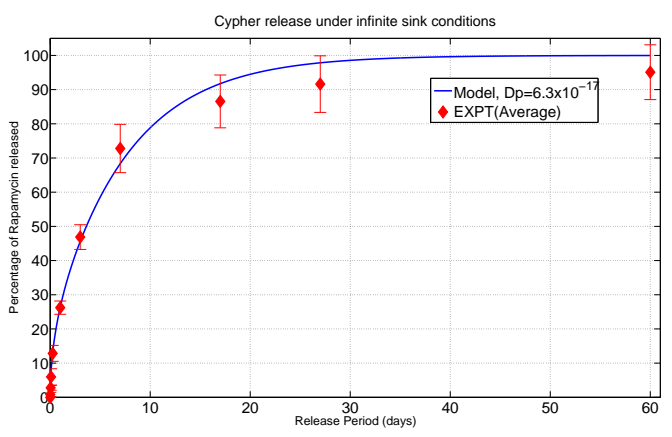

Figure 1: Comparison between in-vitro experimental data and model of Cypher release

and a drug-free topcoat. However, the good fit between the experimental data and the simple model described above suggests that these modelling assumptions are reasonable and capture the release of drug from the Cypher stent, albeit with a layer averaged, or effective diffusion coefficient. A tighter fit is anticipated if the polymer is modelled as a tri-layer system with the drug contained only within the middle layer initially. Actually, it has been shown that the drug can be found in the top coat prior to implantation, suggesting that the intended drug-free top coat may not always be realised. However it is less clear how the in-vivo situation may be modelled. This is considered in the following section.

Newer generation DESs which have focussed on biodegradable polymers and polymer-free modified surface designs have received less attention in the literature in terms of modelling their release. In the case of biodegradable polymeric stents, the drug release is likely governed by diffusion, erosion and possibly dissolution and/or swelling ((Siepmann and Siepmann, 2008); (Fredenberg et al., 2011)). However, in the case of polymer free stents, it is less clear how the sustained release is obtained and how this may be modelled. Furthermore, it even less clear how in-vivo release may be modelled. Realising this, Tzafriri et al. chose to write down a two-part equation to describe drug release from the Cypher and NEVO stents and, using a best fit process, found the values of the parameters of the model (Tzafriri et al., 2012). Their equation assumes that these stents contain two pools of dispersed drug, one that is surface-connected and elutes through a percolating network of drug filled pores, and another that is embedded within the matrix and diffuses more slowly through the percolating polymer phase. Thus they utilised the following equation for the mass of drug released from the Cypher and 
NEVO stents:

$$
M_{\text {stent }}(0)-M_{\text {stent }}(t)=M_{f_{0}}\left(1-e^{-K_{f_{0}} t}\right)+Q_{s u s} \sqrt{t},
$$

where $M_{\text {stent }}(0), M_{f_{0}}, K_{f_{0}}$ and $Q_{\text {sus }}$ denote, respectively, the initial load of drug, the initial pool of first order eluting drug, rate constant and Higuchi rate constant. This equation, however, does not satisfy mass conservation principles: the mass of drug released eventually tends to infinity as time increases. As a result, while their data is well-fitted to this equation for this set of experiments, it is unlikely that their model may be used in a predictive capacity. A further warning must be issued when attempting to identify the release mechanism: it may be possible to fit experimental data to a given model with a variety of different parameter combinations, even if the values of some of the parameters are unlikely (Sirianni et al., 2010).

\section{MODELLING UPTAKE OF DRUG INTO THE ARTERIAL WALL}

While the stent manufacturer may be primarily concerned with stent design and the drug release profile, clinicians are more interested in the drug concentration profile across the arterial wall, and in particular in the target SMCs, as well as the therapeutic duration. Thus an understanding of what happens to the drug in the arterial wall is essential. The arterial wall is porous and plasma flows through the extracellular matrix as a result of the transmural pressure gradient. This pressure gradient induces convection so that drug transport across the arterial wall is governed by a combination of convection, molecular diffusion and binding. A drug with anti-proliferative properties is usually chosen so that when it is uptaken by SMCs it suppresses their proliferation. Depending on the particular properties of the drug, it may also bind to sites within the extracellular matrix.

\subsection{One-dimensional Models}

Early models of drug transport through the arterial wall tended to include only convection and diffusion ((Pontrelli and de Monte, 2007), (Zunino, 2004)) and neglected drug uptake/binding. Furthermore, they restricted the number of dimensions and reduced the problem to a single layer in the arterial wall. One of the first models which encompassed convection, diffusion and uptake into SMC within the porous media was presented by McGinty et al.:

$$
\begin{aligned}
\phi \frac{\partial C_{E}}{\partial t}+v \frac{\partial C_{E}}{\partial x} & =D_{m} \frac{\partial^{2} C_{E}}{\partial x^{2}}-\alpha\left(C_{E}-\frac{C_{I}}{K}\right) \\
(1-\phi) \frac{\partial C_{I}}{\partial t} & =\alpha\left(C_{E}-\frac{C_{I}}{K}\right)
\end{aligned}
$$

where $C_{E}$ and $C_{I}$ denote the volume averaged concentration of drug in the extracellular and cellular regions, respectively (McGinty et al., 2011). The parameters $\phi, v, D_{m}, \alpha$ and $K$ denote the porosity, magnitude of transmural convection, drug diffusion coefficient in the media, drug uptake rate constant and partition coefficient. Equation 4 expresses the rate of uptake of drug by the cells: it is initially proportional to $C_{E}$ but that proportionality diminishes with increasing $C_{I}$ until the carrying capacity (or partition coefficient) of the drug is reached at which point the uptake becomes zero. This system of equations allows for an exchange of drug between the extracellular phase and the cells which is dependent on the concentration in the extracellular phase. They considered the coupled polymer/media system (equations 1,3 and 4) with continuity of drug concentration and continuity of the relative fluxes expressed as boundary conditions at the interface:

$$
\begin{aligned}
C_{p} & =C_{E} \\
-D_{p} \frac{\partial C_{p}}{\partial x} & =-D_{m} \frac{\partial C_{E}}{\partial x}+v C_{E} .
\end{aligned}
$$

They assumed that the flux of drug out of the media was proportional to the concentration at the interface between the media and adventitia to provide the final boundary condition. Their model was also extended to include the adventitia region (where fibroblast cells were modelled in a similar way to SMCs), a topcoat of polymer to slow the release of the drug, and one of the first models of atherosclerotic plaque (modelled using an equilibrium model in the same way as SMCs uptake).

McGinty et al. simulated the problem using a finite difference scheme and conducted a thorough sensitivity analysis which allowed them to infer the importance of the parameters in their model. They found that the results were particularly sensitive to fluctuations in the magnitude of the transmural velocity, and to changes in the drug uptake rate and partition coefficient. Their simple model of plaque suggested that the plaque could act as a reservoir for the drug, ensuring that patients with a higher degree of atherosclerosis may receive therapeutic levels of drug for longer than those with a lesser degree of plaque. This finding is in contrast to an experimental study in the literature (Tzafriri et al., 2010) where it was concluded that drug concentration was inversely correlated to lipid 
concentrations. The McGinty et al. model did, however, neglect the intimal region of the arterial wall and the endothelium layer of cells. Their justification for this is that the endothelium is severely damaged when a stent is inserted and in some cases is completely removed; and indeed the properties of the intimal may not be too different from those in the media.

Despite considering two layers and accounting for convection, diffusion and uptake/binding in each, their model makes several simplifying assumptions. Firstly, the geometry is restricted to one dimension and as such cannot account for the anisotropic nature of diffusion within the tissue ((Levin et al., 2004), (Hwang et al., 2001)). Furthermore, the 1D model clearly has its limitations in approximating $3 \mathrm{D}$ geometry. The strut is considered to be in contact with the arterial wall, when in reality it is likely embedded within it. Finally, the flow interaction problem between the blood and the struts is not accounted for, leading to the potential for under-prediction of drug lost to the lumen, an aspect which has also been investigated in the literature (Zunino, 2004).

Pontrelli and de Monte (Pontrelli and de Monte, 2010) proposed a similar model to that of McGinty et al. which allowed for a diffusion controlled release from the stent as well as convection-diffusionreaction in the arterial wall. Their most sophisticated model has the benefit of being multi-layered, but is unable to model the drug concentration in the SMCs. They do, however, model drug consumption via a linear reaction:

$$
\phi \frac{\partial C_{i}}{\partial t}+2 \gamma_{i} \frac{\partial C_{i}}{\partial x}=D_{i} \frac{\partial^{2} C_{i}}{\partial x^{2}}-\beta_{i} C_{i},
$$

where the subscript $i$ indicates the $i$ th layer and $2 \gamma_{i}$ represents a constant characteristic convection parameter. Pontrelli and de Monte's model has the advantage of admitting an analytical solution.

The convective and diffusive element of the drug transport is well established, as evidenced by the above models and countless others. However, the issue of uptake/binding is more controversial. Some authors have assumed equilibrium models ((McGinty et al., 2011), (Horner et al., 2010), (Abraham et al., 2013)), while others have considered simple loss terms (Pontrelli and de Monte, 2010). More recently, a second order reaction model which allows for saturable reversible binding of sirolimus to specific receptors and general extracellular matrix (ECM) sites has been proposed (Tzafriri et al., 2012). The equations for the rate of uptake of sirolimus to ECM sites and receptors are:

$$
\begin{aligned}
\frac{\partial b_{E C M}}{\partial t}= & k_{o n}^{E C M} c\left(b_{E C M, \max }-b_{E C M}\right) \\
& -k_{o n}^{E C M} k_{d}^{E C M} b_{E C M}, \\
\frac{\partial b_{R E C}}{\partial t}= & k_{o n}^{R E C} c\left(b_{R E C, \max }-b_{R E C}\right) \\
& -k_{o n}^{R E C} k_{d}^{R E C} b_{R E C .} .
\end{aligned}
$$

Here, $c$ is the molar concentration of free drug per unit tissue volume, $b_{E C M}$ and $b_{R E C}$ are the molar concentrations of ECM-bound and receptor-bound drug, respectively. The parameters $b_{E C M, \max }$ and $b_{R E C, \max }$ denote the local molar concentration of ECM and receptor drug binding sites, $k_{o n}^{E C M}$ and $k_{o n}^{R E C}$ are the respective binding on-rate constants and $k_{d}^{E C M}$ and $k_{d}^{R E C}$ are the respective equilibrium dissociation constants. It is certainly true that the drug will bind to binding sites in the tissue and in the cells ((Levin et al., 2005), (Tzafriri et al., 2012), (Bierer et al., 1990)) although the strength of the affinity will likely vary substantially with the particular drug under consideration. Furthermore, it is not clear how the density of the binding sites could easily be determined. Thus it may be that this binding model is specific to a particular class of drugs and not suitable for more general compounds. A greater understanding of the binding process would undoubtedly assist with model development.

\subsection{Higher-dimensional Models}

Despite providing useful, and in some cases, counterintuitive physiological insights, one-dimensional models are inadequate for accurately resolving quantitative aspects. When the dimension of the model is increased, numerical approaches are necessarily required.

Two-dimensional models in simplified geometries were computed by (Hwang et al., 2001), (Grassi et al., 2009) and (Zunino, 2004) among others. A number of three-dimensional models have also been devised. (Weiler et al., 2012) provided a broad generalization of the works of (Mongrain et al., 2007), (Zunino et al., 2009) and (Vairo et al., 2010); a three-dimensional model of drug transport in the lumen and the arterial wall. Laminar steady flow was assumed in the lumen and the steady diffusion equation (no convection) in the arterial wall. Through numerical simulation using commercial finite element software, they found that the highest rates of mass transfer occurred at the forward portion of the stent and the rate of drug delivery to the lumen was greater than that to the tissue.

(Horner et al., 2010) appear to be one of the first authors to provide a three-dimensional reaction- 
diffusion-convection model in a realistic geometry. They stress the importance of considering two phases of the drug (bound and unbound) and use a first order reaction kinetics model to describe the transfer of drug between the two phases. They utilise ABAQUS to obtain a realistic geometry of a deformed stent and vessel wall and then utilise FLUENT to solve their transport equations. Their three-dimensional setting allows for the consideration of anisotropic diffusion in the arterial wall. They do, however, make three significant simplifications. Firstly, they model the arterial wall as a linear homogeneous solid and do not distinguish between the intima, media and adventitia. Secondly, despite calculating the transmural velocity field, they assume this is fixed when solving the transport equations. Perhaps the most unrealistic assumption is that the drug concentration on the stent remains constant and does not deplete. They find that deposition patterns tend to follow the pattern of the stent struts and that the drug is able to penetrate deep into the arterial wall. The pattern of bound drug becomes less uniform as the Peclet number is increased, eventually becoming restricted to areas adjacent to the struts, as convection dominates over diffusion.

\section{EXPERIMENTAL VALIDATION}

An important aspect of modelling and simulation is validation. The accuracy of the model results can only ever be as good as the quality of the inputs, especially when the model is sensitive to changes in one or more of the parameters. At present, before a DES is approved for use in humans it must undergo in-vivo testing in an animal model (for example porcine coronary artery); this is a very costly exercise and also raises ethical questions. However, a series of in-vitro and ex-vivo experiments can be carried out at various stages of the modelling process to verify certain aspects and to suggest improvements/modifications to the model.

One such example of this is in the estimation of the model parameters. In our laboratory we have been performing in-vitro DES release experiments which have allowed us to estimate the diffusion coefficient of the drug in polymer-coated stents based on a least squares analysis and utilising analytical solutions (Figure 1). The experiments consisted of placing Cypher DESs in a sealed glass vial containing physiological release medium (phosphate buffered saline:ethanol (90:10)). At several time points up to 60 days, the stent was removed and placed in a separate vial containing fresh release medium, with the mass of drug in the original solution subsequently quantified using UV-spectroscopy. These simple experiments give confidence in the modelling and provide reliable estimates of the drug diffusion coefficient in the polymer, which can feed into more sophisticated models. Alternatively, a diffusion cell containing a membrane made from the polymer under study can be used. The diffusion coefficients in each layer of the arterial wall can be measured in a similar way. The porosity of each layer of the arterial wall may be measured from histological sections by quantitative microscopy. Drug uptake/binding parameters, such as the partition coefficient $K$ and uptake rate constant $\alpha$ in equations (3-4) may be estimated by quantifying drug uptaken by cells grown in culture plates at different time points. For estimation of the parameters in the second order reaction model (equations 8-9), the reader is referred to (Tzafriri et al., 2012) and references therein.

But the use of experiments to inform the modelling is not a one-way process. Indeed, our group have utilised the modelling to design experiments which in turn have fed back into the model. For example, recognising that one of the important features of in-vivo drug release from DESs is transport by convection, in our laboratory we are developing ex-vivo perfusion circuit experiments which will allow us to control the intraluminal pressure (and thus vary convection) across the arterial wall. Thus, experimental validation should not be seen as the final step, only to be performed once the model has been built and the results simulated. Instead, the modelling and experimentation should go hand-in-hand, complementing each other.

\section{MODELLING CONSIDERATIONS FOR THE FUTURE}

Whilst significant progress has been made in simulating various aspects of drug-eluting stents, there is still a need to better understand the drug elution process and the drug transport in the lumen and arterial wall. Here we indicate some possible modelling considerations for future research in this field so that the aim of achieving an 'optimal' drug-eluting stent may be realised.

\subsection{Simulating Drug Release from New Generation DESs}

There is a real need for models to be developed for drug release from newer generation biodegrad- 
able polymer coated stents and polymer-free surfacemodified stents. It may be that the dominant release mechanism in these stents is not diffusion and so models which assume purely diffusion may not capture the release kinetics. Any new models should be experimentally validated in-vitro to verify the physical processes governing the release have been captured. It is anticipated that for most DESs the in-vivo release profile may be significantly different due to the complex biological processes involved, but if the in-vitro release can be well modelled then this should shed some light as to how to model the in-vivo situation. Comparison of the in-vitro drug release profile of different stent platforms may indicate the release mechanism(s) which give rise to the most favourable release profile. This, in turn, may allow for better design of DESs in the future.

\subsection{Inclusion of the Endothelium, Intima and Plaque}

The inner layer of the the arterial wall, the intima, is a thin layer comprising the endothelium as well as the elastic lamina. It is well established that the endothelium is damaged during the stent insertion process and in some cases even removed. As a result, many current models neglect the intima region.

A more complete model of the arterial wall should include the intima region as well as the endothelium and internal elastic lamina. The endothelium is important in vasoconstriction and vasodilation and regulates the uptake of plasma into the arterial wall. While diseased endothelium is dysfunctional (this is the starting point of atherosclerosis) it will nevertheless have an effect on the relative importance of convection to diffusion in the arterial wall. It is anticipated that drug transport through the initma will occur via similar processes to that in the media, namely diffusion (albeit with a different diffusion coefficient from the media), convection and possibly binding.

Another possible modelling consideration is atherosclerotic plaque; the presence of the plaque is the very reason that the stent is inserted and yet it has received very little attention in the literature. McGinty et al. seem to be the only authors who have attempted to model the plaque to date, although (Tzafriri et al., 2010) have experimentally examined the effect of the plaque. The plaque is known to contain a fibrous cap of variable thickness as well as a necrotic core made up of cellular debris, cholesterol cleft and cell membranes. Furthermore, the plaque also contains macrophages and SMCs as well as a lipid pool containing lipid dispersed in a collagen matrix. A More sophisticated model of plaque, tak- ing into account its various components, may provide more insight into the effect of plaque on tissue drug concentrations.

\subsection{Modelling Lumenal Blood Flow and Stent Interaction}

In simulations where the blood flow is taken into account, it is common for the blood flow to be modelled as steady Poiseuille flow. Of course, in reality blood flow near the heart is pulsatile and the artery is continually contracting and expanding. The presence of the stent interrupts the flow (Peacock et al., 1995) and the effect this has on the drug transport should be simulated.

In addition to simply considering some of the parameters to be time and space dependent, it may be necessary to consider the proliferation of SMCs and neointima growth as a wound healing problem.

\subsection{Inclusion of Complex Three-Dimensional Geometry}

While simplified one-dimensional models can provide useful insights into this problem, ultimately three-dimensional models which capture the full complex geometry of the stent and the arterial wall are required. The idea of numerically simulating such a complex problem may have seemed impossible not so long ago, but with the accelerating advances in computational power and numerical techniques it is now possible. The existing three-dimensional models in the literature all make certain simplifying assumptions, whether it be in idealising the stent geometry, or in neglecting convection, diffusion or binding, or in considering only single or bi-layer arterial walls. Thus there is an opportunity to increase the sophistication of the three-dimensional models, whether it be incrementally or in one fell swoop. However, caution must be exercised to ensure that the results of the simulations are not subject to high uncertainty, in which case the fidelity of the results may be called into question.

\section{CONCLUSIONS}

Drug-eluting stents have significantly improved the treatment of coronary artery disease. Despite real progress being made in the past decade, a complete understanding of DESs is still some way off. The process of in-vitro drug release from newer generation DESs is still not fully understood, let alone the 
complex in-vivo situation where flowing blood, pulsatility, wound healing, proliferation and migration of SMCs and complex uptake/binding no doubt all play some part. Future research should include the modelling of drug release from biodegradable and polymer-free modified surface stents, more accurately modelling lumenal blood flow and stent interaction, including the endothelium, the intima and atherosclerotic plaque. In order to be able to use simulations in a predictive capacity, three-dimensional models which encompass the full complex geometry are necessarily required. But care should be taken to verify the correctness of the numerical results. Finally, the modelling should be complemented by appropriate experiments to validate the resulting simulations and improve on the model.

\section{ACKNOWLEDGEMENTS}

We would like to acknowledge the funding provided by EPSRC under grant number EP/J007242/1. The first author would also like to acknowledge the receipt of a Carnegie Scholarship.

\section{REFERENCES}

Abraham, J. P., Gorman, J. M., Sparrow, E. M., Stark, J. R., and Kohler, R. E. (2013). A mass transfer model of temporal drug deposition in artery walls. Int. J. Heat Mass Trans., 58:632-638.

Beers, M. H. (2004). The Merck Manual of Health \& Aging. Elsevier Health Sciences, London.

Bierer, B. E., Patilla, P. S., Standaert, R. F., Herzenberg, L. A., Burakoff, S. J., Crabtree, G., and Schreiber, S. (1990). Two distinct signal transmission pathways in $t$ lymphocytes are inhibited by complexes formed between an immunophilin and either fk605 or rapamycin. Proc. Natl. Acad. Sci. USA, 87:9231-9235.

Delfour, M. C., Garon, A., and Longo, V. (2005). Modeling and design of coated stents to optimize the effect of the dose. SIAM J. Appl. Math.., 65(3):858-881.

Fredenberg, S., Wahlgren, M., Reslow, M., and Axelsson, A. (2011). The mechanisms of drug release in poly(lactic-co-glycolic acid)-based drug delivery systems - a review. Int. J. Pharmaceutics, 415:34-52.

Garasic, J. M., Edelman, E. R., Squire, J. C., Seifert, P., Williams, M. S., and Rogers, C. (2000). Stent and artery geometry determine intimal thickening independent of arterial injury. Circulation, 101(7):812818.

Grassi, M., Pontrelli, G., Teresi, L., Grassi, G., Comel, L., Ferluga, A., and Galasso, L. (2009). Novel design of drug delivery in stented arteries: a numerical comparative study. Math. Biosci. Eng., 6(3):493-508.

Horner, M., Joshi, S., Dhruva, V., Sett, S., and Stewart, S. F. C. (2010). A two-species drug delivery model is required to predict deposition from drug-eluting stents. Cardiovasc. Eng. Technol., 1(3):225-234.

Hwang, W., Wu, D., and Edelman, E. R. (2001). Physiological transport forces govern drug-distribution for stent based delivery. Circulation, 104(7):600-605.

Khan, W., Farah, S., and Domb, A. J. (2012). Drug eluting stents: Developments and current status. J. Controlled Release., 161:703-712.

Levin, A. D., Jonas, M., Hwang, C. W., and Edelman, E. R. (2005). Local and systemic drug competition in drug-eluting stent tissue deposition properties. $J$. Controlled Release, 109:236-243.

Levin, A. D., Vukmirovic, N., Hwang, C. W., and Edelman, E. R. (2004). Specific binding to intracellular proteins determines arterial transport properties for rapamycin and paclitaxel. Proc. Natl. Acad. Sci. USA, 101(25):9463-9467.

Lloyde-Jones, D. (2010). Heart disease and stroke statistics2010 update: A report from the american heart association. Circulation, 121:e46-e215.

Lusis, A. (2000). Atherosclerosis. Nature, 407:233-241.

McGinty, S., McKee, S., Wadsworth, R. M., and McCormick, C. (2011). Modelling drug-eluting stents. Math. Med. Biol., 28:1-29.

Mongrain, R., Faik, I., Leask, R., Rodes-Cabau, J., Larose, E., and Bertrand, O. (2007). Effects of diffusion coefficients and struts apposition using numerical simu- 
lations for drug eluting coronary stents. J. Biomech. Eng., 129:733-742.

Murray, C. and Lopez, A. (1997). Alternative projections of mortality and disability by cause 1990-2020: Global burden of disease study. The Lancet, 349(9064):14981504.

Peacock, J., Hankins, S., Jones, T., and Lutz, R. (1995). Flow instabilities induced by coronary artery stents: Assessment with an in vitro pulse duplicator. $J$. Biomech, 28:17-26.

Pontrelli, G. and de Monte, F. (2007). Mass diffusion through two-layer porous media: an application to the drug-eluting stent. Int J. Heat Mass Trans., 50:36583669 .

Pontrelli, G. and de Monte, F. (2010). A multi-layer porous wall model for coronary drug-eluting stents. Int $J$. Heat Mass Trans., 53:13629-3627.

Siepmann, J. and Siepmann, F. (2008). Mathematical modelling of drug delivery. Int. J. Pharmaceutics, 364:328-343.

Sirianni, R. W., Jang, E.-H., Miller, K. M., and Saltzman, W. M. (2010). Parameter estimation methodology in a model of hydrophobic drug release from a polymer coating. SIAM J. Appl. Math., 142):474-482.

Stefanini, G. G. and Holmes, D. R. (2013). Drug-eluting coronary artery stents. N. Engl. J. Med., 368:254-265.

Tambaca, J., Kosor, M., Canic, S., and Paniagua, D. (2010). Mathematical modeling of vascular stents. SIAM J. Appl. Math., 70(6):1922-1952.

Tzafriri, A., Vukmirovic, N., Kolachalama, V., Astafieve, I., and Edelman, E. R. (2010). Lesion complexity determines arterial drug distribution after local drug delivery. J. Controlled Release, 142(3):332-338.

Tzafriri, A. R., Groothuis, A., Price, G. S., and Edelman, E. R. (2012). Stent elution rate determines drug deposition and receptor-mediated effects. J. Controlled Release, 161:918-926.

Vairo, G., Cioffi, M., Cottone, R., Dubini, G., and Migliavacca, F. (2010). Drug release from coronary artery stents: a multidomain approach. J. Biomech., 43:1580-1589.

Weiler, J. M., Sparrow, E. M., and Ramazani, R. (2012). Mass transfer by advection and diffusion from a drugeluting stent. J. Heat Mass Transfer, 55:1-7.

Zunino, P. (2004). Multidimensional pharmacokinetic models applied to the deign of drug-eluting stents. Cardiov. Eng.: Int. J., 4(2):181-191.

Zunino, P., D’Angelo, C., Petrini, L., Vergara, C., Capelli, C., and Migliavacca, F. (2009). Numerical simulation of drug eluting coronary stents: mechanics, fluid dynamics and drug release. Comput. Methods Appl. Mech. Eng.., 198:3633-3644. 